\title{
Ruptured Gall Bladder containing Stones following Blunt Trauma Abdomen: A Rare Presentation of Hemodynamic Instability
}

\author{
Vineet Goel, ${ }^{1}$ Naveen Kumar, ${ }^{1}$ Nikhil Soni ${ }^{1}$ \\ 'Department of Surgery, Hindu Rao Hospital, Ashok Vihar, Delhi, India.
}

\section{ABSTRACT}

Gall bladder injuries are seen in $2 \%$ of patients undergoing laparotomy for blunt trauma abdomen. Isolated gall bladder injury is a rare event with associated presence of stones is even rarer. The associated visceral injuries lead to intraoperative identification in most cases. Here we present a case of 30 years old male with isolated gall bladder laceration following blunt abdominal trauma. The diagnosis of gallbladder perforation after blunt injury may be suspected in patients with signs of an acute abdomen and hypotension that is not explained by blood loss. Early suspicion and prompt exploration is imperative. Cholecystectomy is an adequate treatment for the condition.

Keywords: acute abdomen; cholecystectomy; hypotension; laparotomy.

\section{INTRODUCTION}

Extra-hepatic injuries in a blunt trauma patient are an uncommon finding. ${ }^{1-4}$ Gall bladder is a well-protected organ being embedded in liver and covered by rib cage. Consequently isolated gall bladder injuries are seen less often. Rupture of thickened gall bladder with stones is a rarity as thin walled or distended organ at the time of injury is considered as a prerequisite as seen after meals or alcohol intoxication. ${ }^{1}$ The site of injury, abdominal findings and unexplained hemodynamic instability must lead to suspicion of extra hepatic biliary injury and early exploration is warranted. ${ }^{3}$

\section{CASE REPORT}

A 30 year old male presented to surgical emergency after referral from other hospital with a history of road traffic accident and right upper abdominal trauma four hours back. On presentation he had pain in upper abdomen with multiple episodes of vomiting. On examination his pulse was $120 / \mathrm{min}$ and blood pressure $80 / 40 \mathrm{~mm}$ of $\mathrm{Hg}$. Immediate fluid resuscitation was started following which blood pressure increased to $100 / 70 \mathrm{~mm}$ of hg after 30 min of resuscitation. On abdominal examination, diffuse tenderness and muscle guarding was present but without abdominal distension. Laboratory studies were within normal limits. Chest $\mathrm{x}$-ray was normal. FAST was positive with fluid pockets in sub-hepatic and pelvic spaces. Patient's blood pressure decreased again to $90 / 60 \mathrm{~mm}$ of $\mathrm{Hg}$ after one hour of presentation during monitoring.

In view of FAST being positive and hemodynamic instability, no further imaging was done. The provisional clinical diagnosis of liver laceration with hemo-peritoneum was made and immediate exploratory laparotomy was performed which revealed gall bladder perforation of size $2 \times 1 \mathrm{~cm}$ in fundus region (Figure 1). Multiple gall stones were found to be spilled in the peritoneal cavity. Leakage of bile and bleeding from gall bladder wall was

Correspondence: Dr. Naveen Kumar, Department ff Surgery, Hindu Rao Hospital, B-33 Phase 4, MIG flats, Ashok Vihar, Delhi, India. Email: dr.naveenms@gmail.com, Phone: +91-9873651811. 
noted. $200 \mathrm{ml}$ of bilio-sanguinous fluid was aspirated from peritoneal cavity. Liver, stomach, adjacent bowel and viscera revealed no injury. Cholecystectomy with peritoneal lavage was performed. Post-operative period was uneventful and patient got discharged on day five.

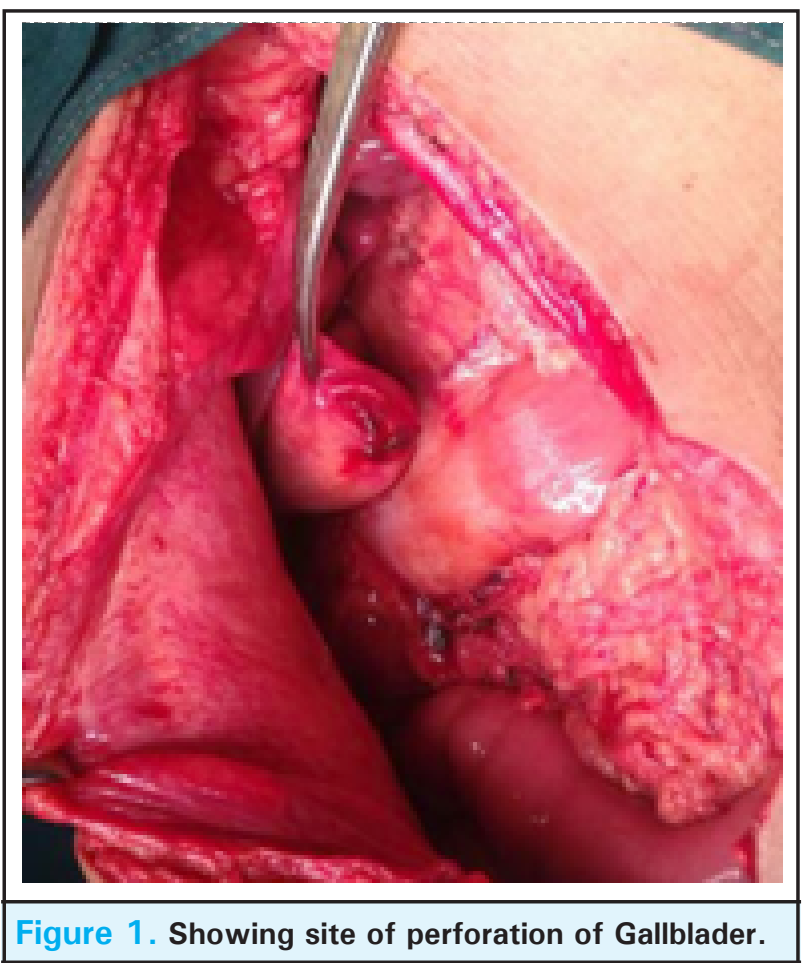

\section{DISCUSSION}

Gall bladder injuries are most often seen following penetrating abdominal trauma. ${ }^{5}$ Extra hepatic injuries after blunt trauma are mostly accompanied with liver injuries (approximately $83 \%$ cases) or visceral injuries. ${ }^{1}$ Only $2 \%$ of patients undergoing laparotomy for blunt trauma have gall bladder injuries. Isolated extra hepatic injuries particularly gall bladder injuries are rare. ${ }^{1}$ In his review of literature Chad et al found only 45 gall bladder injuries out of 40,000 injured patients attending level I trauma center [6]. Even rarer is a rupture of gall bladder containing stones as in our case. After reviewing the published literature we could only find two reported cases of such a scenario. ${ }^{1}$

Earliest classification by Penn et al classified four major groups of gallbladder injury as contusion, avulsion, laceration and traumatic cholecystitis. ${ }^{2}$ Contusion, defined as intramural hematoma, is most often diagnosed at the time of laparotomy may lead to delayed perforation due to wall necrosis Perforation, also known as "rupture" or "laceration," is the most commonly reported injury as in this case. Avulsion can be partial avulsion, in which the gallbladder is partially torn from the liver bed; complete avulsion, in which the gallbladder is completely separated from the liver but with intact cystic duct and artery; and total avulsion or traumatic cholecystectomy. ${ }^{7}$ Losanoff and Kjossev describe a more detailed classification of blunt gallbladder injuries; according to their classification, our patient belongs to type 2 (rupture of gall bladder). ${ }^{8}$ Due to rarity of the condition exact incidence of various types of injury doesn't exist in published literature.

Gall bladder injuries are predisposed by factors such as thin-walled normal gallbladder than a diseased thick one, a distended gall bladder, and alcohol consumption that increases the tone of sphincter of Oddi and biliary tract pressure. It is postulated that gallbladder distention at the time of trauma is a prerequisite for its rupture. ${ }^{1}$

According to most of the published data the clinical presentation of extra hepatic biliary injuries remain variable and nonspecific, resulting in a delay in diagnosis. ${ }^{1}$ Though persistent bile leakage causes intra-peritoneal bilious collections, some patients develop early chemical peritonitis while most develop it later. Both conditions, however, require aggressive surgical intervention, since continuous bile loss into the abdomen has high morbidity and mortality. ${ }^{1}$

Early diagnosis of gallbladder injury due to blunt abdominal trauma is essential but is difficult to establish. ${ }^{1}$ Site and mechanism of injury, hypotension and signs of acute abdomen may aid in diagnosis [3]. Diagnostic procedures are not always helpful. Peritoneal tap or lavage may reveal bile-stained fluid, yet has a high incidence of false-negative results. ${ }^{1}$ On ultrasound, non-visualization of gall bladder raises the suspicion of gall bladder avulsion or rupture. Though CT scan is considered 'gold standard' to diagnose such injuries but study is non-specific. Signs of gallbladder injury are pericholecystic fluid, an ill-defined contour of the gallbladder wall, a mass effect on the duodenum, highattenuation intraluminal material (blood), a thickened gallbladder wall, and a collapsed gallbladder in a fasting patient. ${ }^{10}$

The treatment of gall bladder injuries is cholecystectomy as supported in literature. ${ }^{1,3,6}$ However, less severe injuries like contusions and partial avulsions can sometimes be observed. ${ }^{4,7}$ In our case early presentation of patient with hemodynamic instability and acute abdominal signs prompted decision for exploratory laparotomy and subsequent cholecystectomy with uneventful post-operative recovery for the patient.

Isolated gall bladder injuries are rare. High degree of suspicion should be kept and should prompt surgeons for rapid work-up and subsequent treatment of the patient to prevent delay in diagnosis and eventual 
fatality. The prognosis is good if treatment is started early and there is no other serious injury as in our patient. Cholecystectomy is an adequate treatment for the condition.

\section{ACKNOWLEDGEMENTS}

Department of Surgery, Hindu Rao Hospital, Delhi, India.

\section{REFERENCES}

1. Schachter P, Czerniak A, Shemesh E, Avigad I, Lotan G, Wolfstein I. Isolated gallbladder rupture due to blunt abdominal trauma. HPB Surg. 1989;1(4):359-62.

2. Penn I. Injuries to the gallbladder. Br J Surg.1966;49:636-41.

3. Wiener I, Watson LC, Wolma FJ. Perforation of the gallbladder due to blunt abdominal trauma. Arch Surg. 1982 Jun;117(6):805-7.

4. Soderstrom CA, Maekawa K, Du Priest RW Jr, Cowley RA. Gallbladder injuries resulting from blunt abdominal trauma. Ann Surg. 1981 Jan;193(1):60-6.

5. Salzman S, Lutfi R, Fishman D, Merlotti G. Traumatic rupture of the gallbladder. J Trauma. 2006 Aug;61(2):454-6.
6. Ball CG, Dixon E, Kirkpatrick AW, Sutherland FR, Laupland $\mathrm{KB}$, Feliciano DV. A decade of experience with injuries to the gallbladder. J Trauma Manag Outcomes. 2010 Apr 15;4:3.

7. Sharma O. Blunt gall bladder injuries: presentation of twenty-two cases with review of the literature. J Trauma. 1995 Sep;39(3):576-80.

8. Losanoff JE, Kjossev KT. Complete traumatic avulsion of the gallbladder. Injury. 1999 Jun;30(5):365-8.

9. Chen X, Talner LB, Jurkovich GJ. Gallbladder avulsion due to blunt trauma. AJR Am J Roentgenol. 2001 Oct;177(4):822.

10. Pavlidis TE, Lalountas MA, Psarras K et al. Isolated complete avulsion of the gallbladder (near traumatic cholecystectomy): a case report and review of the literature. J Med Case Rep. 2011 Aug 18;5:392. 